\title{
Patterning stem cells: What we learn from the feather regeneration model
}

\author{
Ting Xin Jiang ${ }^{1}$, Zhi Cao Yue ${ }^{1}$, Ping Wu ${ }^{1}$, Cheng Ming Chuong ${ }^{1}$ \\ ${ }^{I}$ Department of Pathology, University of Southern California
}

Stem cells have the remarkable potential to develop into many different cell types in a well organized spatial pattern. How adult stem cells regenerate and pattern themselves are mostly unknown. Chicken feather is a good model for studying regeneration because feathers undergo repetitive molting and regeneration under physiological conditions. The epithelial cells are laid out in an exquisite hierarchical branching pattern, down to single cell. It is a highly sensitive assay because any perturbation of the patterning process can be revealed in this high resolution model.

Here we identify feather follicle stem cells with long-tern label-retaining (LRCs). LRCs are located in a collar bulge niche and configured as a ring which is horizontally placed in radially symmetric feathers but tilted in bilaterally symmetric feathers. They have a high efficiency of incorporation into developing skin and can form different components of feather follicles (Yue et al., 2005). An anterior - posterior Wnt3a gradient was found in bilateral symmetric flight feathers, but not radial symmetric downy feathers. Flattening out wnt 3a gradient converts bilateral to radial symmetric feathers. Transplantation experiments showed that different micro-environments for the stem cell are dictated by dermal papilla (Yue et al., 2006). Currently we are characterizing more of this microenvironment surrounding epidermal stem cells.

How was the feather follicles set up to begin with? During early feather bud formation, we can dissociate mesenchyme cells and reconstitute them in vitro. They can reform feather bud and interbud cells. Changing the environments can lead to different number, size and spacing of feather buds. This high resolution model help reveal principles in stem cell patterning. It offers new understanding in stem cell biology and morphogenesis, and may lead to new clues to human organ regeneration in the future.

Keywords: regeneration, pattern formation, Wnt, epidermal stem cells, dermal papilla

Cell Research (2008) 18:s70. doi: 10.1038/cr.2008.160; published online 4 August 2008

Correspondence: Ting Xin Jiang.

E-mail: tjiang@usc.edu

Lab website: http://www-hsc.usc.edu/ cmchuong

Jiang TX, Jung HS, Widelitz RB, Chuong CM. Self organization of periodic patterns by dissociated feather mesenchymal cells and the regulation of size, number and spacing of primordia. Development 1999;
126:4997-5009.

Yue Z, Jiang TX, Widelitz RB, Chuong CM. Mapping stem cell activities in the feather follicle. Nature 2005; 438:1026-1029.

Yue Z, Jiang TX, Widelitz RB, Chuong CM. Wnt 3a gradient converts radial to bilateral feather symmetry via topological arrangement of epithelia. Proc Natl Acad Sci USA 2006; 103:951-955. 